\title{
Groove resection of pancreatic head in groove pancreatitis: A case report
}

\author{
CHUNFU ZHU ${ }^{1}$, QIN HUANG ${ }^{2}$, JIANFEI ZHU ${ }^{1}$, XUDONG ZHANG $^{1}$ and XIHU QIN ${ }^{1}$ \\ ${ }^{1}$ Department of Hepato-biliary-pancreatic Surgery, Changzhou No. 2 People's Hospital, Nanjing Medical \\ University, Changzhou, Jiangsu 213000, P.R. China; ${ }^{2}$ Department of Pathology and Laboratory Medicine, \\ Veterans Affairs Boston Healthcare System, Harvard Medical School, West Roxbury, MA 02132, USA
}

Received June 6, 2016; Accepted April 20, 2017

DOI: $10.3892 /$ etm.2017.4753

\begin{abstract}
Groove pancreatitis (GP) is a rare type of chronic pancreatitis, which primarily affects the groove area of the pancreatic head. Surgery is considered as the ultimate method for GP if the symptoms are not improved by conservative or endoscopic treatments, and the Whipple technique is the common choice at present. The present study introduces a novel surgical procedure for GP, namely groove resection of pancreatic head (GRPH), in a 56-year-old male patient. To the best of our knowledge, this is the first report on GRPH for the surgical treatment of GP. This patient was diagnosed with typical GP without any evidence of malignancy, based on imaging studies and gastrointestinal endoscopy, and was treated with GRPH. Briefly, only the groove area of the pancreatic head was resected, while the duodenum, the main pancreatic duct and the majority of the pancreatic head were preserved. A complete relief of abdominal pain and a weight gain were achieved 2 months after surgery. Endoscopic examination indicated marked improvement of the duodenal lesions. In conclusion, GRPH is a less invasive surgical procedure for GP compared with other surgical procedures and may be an alternative for the surgical treatment of GP without severe lesions or stenosis of the duodenum.
\end{abstract}

\section{Introduction}

The pancreatic duodenal groove is an anatomical area confined between the duodenum, distal common bile duct and pancreatic head (1). Groove pancreatitis (GP), which was first described by Becker in 1973 (2), refers to a rare type of chronic pancreatitis that primarily affects the groove area of the pancreas $(1,3)$. The pathogenesis of GP remains unclear, and a specific treatment

Correspondence to: $\mathrm{Dr}$ Xihu Qin, Department of Hepato-biliary-pancreatic Surgery, Changzhou No. 2 People's Hospital, Nanjing Medical University, 29 XingLongXiang Road, Changzhou, Jiangsu 213000, P.R. China

E-mail: xihuqin11@sina.com

Key words: pancreas, groove pancreatitis, groove resection strategy has yet to be established (4). The reported treatments of GP include medical $(3,4)$, endoscopic (5) or surgical approaches $(3,4,6-8)$, according to the clinical features of the condition. The main drawback of nonsurgical approaches is the high risk of recurrence of symptoms subsequent to treatment cessation $(5,9)$. Therefore, surgical treatment is considered a good choice for GP when the symptoms are not significantly improved or are recurrent following medicine or endoscopic treatment, or in cases with a suspicion of malignancy. Surgical methods reported in the literature include cephalic pancreaticoduodenectomy (PD; also known as the Whipple technique) $(6,7)$, duodenum-preserving pancreatic head resection (DPPHR) $(3,4,8)$, pancreas-preserving duodenal resection (PPDR) (4), or a simple draining procedure (10). The present study reported the use of a novel surgical procedure for GP, known as groove resection of pancreatic head (GRPH), which is less invasive compared with other surgical methods.

\section{Case report}

Written informed consent was obtained from the patient prior to participation in the present study. A 56-year-old male patient was admitted to in March 2015 to Changzhou No. 2 People's Hospital (Changzhou, China) due to recurrent upper abdominal pain and poor appetite that persisted for $\sim 1$ month, accompanied by a weight loss of $3 \mathrm{~kg}$. The patient had several admissions at various hospitals due to repeated abdominal pain over the past 3 years. This patient had a history of alcohol abuse of 300 grams per day for 20 years, and a smoking history of 40-60 cigarettes a day for 30 years. No vomiting or nausea were reported, and no history of diabetes mellitus, hypertension or prior abdominal surgery.

A physical examination demonstrated mild tenderness in the upper abdomen and periumbilical region, while no abdominal mass was detected. Routine blood test revealed normal white blood cell, red blood cell and platelet counts. Serologic testing demonstrated that the levels of total bilirubin, direct bilirubin, alanine aminotransferase, aspartate aminotransferase, alkaline phosphatase, amylase, triglyceride, total cholesterol, urea nitrogen and creatinine were within the normal range. The levels of blood glucose and glycated hemoglobin were normal. Total serum protein level was $52.2 \mathrm{~g} / 1$ (normal range, 68-82 $\mathrm{g} / \mathrm{l}$ ) and albumin level was 
$32.9 \mathrm{~g} / \mathrm{l}$ (normal range, 35-50 g/l). A tumor marker test indicated that the level of carbohydrate antigen 19-9 (CA19-9) was $49.47 \mathrm{U} / \mathrm{ml}$, which was slightly elevated (reference range, 0-45 U/ml). The CA50, CA125, carcinoembryonic antigen and $\alpha$-fetoprotein levels, as well as serum IgG4 and the ratio of $\mathrm{IgG} 4$ to $\mathrm{IgG}$, were all normal.

A computed tomography (CT) scan revealed a hypodense lesion in the groove area of pancreatic head (Fig. 1A-C). Thickening of the wall and cystic formation on the second part of the duodenum were observed (Fig. 1D-F). The CT findings were further confirmed by magnetic resonance imaging (MRI) examination (Fig. 1G-I). An accessory pancreatic duct was not demonstrated on CT or MRI scans, while a large right renal cyst was observed on CT and MRI (Fig. 1). In addition, a magnetic resonance cholangiopancreatography (MRCP) examination indicated mildly dilated proximal common bile duct and slight narrowing of the distal bile duct (DBD; Fig. 2). Mildly dilated main pancreatic duct was also presented on the MRCP image (Fig. 2). Upper gastrointestinal endoscopy revealed edematous mucosa and multiple polypoid protuberances in the first and second portions of the duodenum (Fig. 3A). No luminal narrowing of the duodenum was identified by endoscopy (Fig. 3B). Furthermore, histological examination (hematoxylin and eosin staining) of the polypoid biopsy specimen showed chronic and active mucosal inflammation and edema in the mesenchyme (Fig. 3C). The major papilla had a normal appearance and no minor papilla was found. There was no mucosal erosion or ulceration in the stomach or duodenum.

All these findings appeared consistent with the diagnosis of GP, and there was no evidence of malignancy. The patient was then treated with proton pump inhibitors, a pancreatic enzyme supplement and analgesics, as well as abstinence from alcohol and tobacco. After 2 weeks, the patient complained of no significant improvement of clinical symptoms. Exploratory laparotomy was considered following a thorough discussion with the patient and his family.

The exploratory laparotomy was performed under general anesthesia (initiated with $5 \mathrm{mg}$ imidazole valium, $20 \mathrm{mg}$ etomidate, $30 \mu \mathrm{g}$ sufentanil, $50 \mathrm{mg}$ rocuronium bromide, and maintained with $250-300 \mathrm{mg} / \mathrm{h}$ propofol, 200-300 $\mu \mathrm{g} / \mathrm{h}$ fentanyl, and $5 \mathrm{mg} / \mathrm{h}$ cisatracurium). No ascites were detected in the abdominal cavity. The gastrocolic ligament was adherent to the pancreas and duodenum, and was ligated to fully expose the front side of the pancreas and the duodenum. Kocher maneuver was performed to expose the back region of the duodenum and pancreatic head. The Treitz fusion fascia, located at the back of the duodenum and pancreatic head, was carefully preserved. The first and second portions of the duodenum were infiltrated with fibrotic tissues around them, but no mass or deformation was observed on the duodenal wall (Fig. 4A). The groove area is exhibited in Fig. 4A, and this appeared as a narrowing area in the right part of the pancreatic head with a groove-like shape. As shown in Fig. 4A, the surface of the groove area was irregularly ridged and hard. The range of the groove area was marked for GRPH.

Initially, the loose connective tissues and blood vessels between the pancreatic head and duodenum were dissociated (Fig. 4B). Next, the lower part of the pancreatic head was divided and the superior mesenteric vein (SMV) was exposed. The pancreatic tissue was divided along the left side of the groove area, preserving the pancreatic tissue between the SMV and the groove (Fig. 4C). The inferior part of the groove pancreas was lifted upward, and the division was continued along the posterior border of the groove (Fig. 4D). In order to intraoperatively identify the DBD, the gallbladder was resected and a catheter was inserted into the DBD via the cholecystic duct. By tracing the catheter, DBD was confirmed and isolated carefully near the papilla of Vater. The separation was advanced cranially along the front surface of the DBD (Fig. 4E). Finally, the upper part of the groove pancreas was divided along the anterosuperior border, which was the first portion of the duodenum. The gastroduodenal artery and the posterior superior pancreaticoduodenal artery were not shown intraoperatively, but were preserved in the current case. After the groove pancreas was resected and removed, a defect was observed in the shape of a narrowing groove (Fig. 4F). The resected specimen was approximately $5 \times 3 \mathrm{~cm}$ in size (Fig. 5A). Subsequent to cutting longitudinally, a white mass was shown with a size of $2 \times 2.5 \mathrm{~cm}$ and a clear boundary (Fig. 5A). No accessory pancreatic duct was observed in the residual pancreatic tissues and the specimen. An intraoperative pathological examination of the specimen indicated acute inflammatory cell infiltration, edema and congestion of the pancreatic parenchyma, and evident fibrosis of the pancreatic mesenchyme in the groove area. No calcification or protein plugs were observed, and there was no evidence of malignancy. A postoperative routine pathological examination with hematoxylin and eosin staining for $5 \mathrm{~min}$ at room temperature (Fig. 5B) confirmed the diagnosis of GP. Digital images were obtained using a DP Manager and a light microscope (Olympus Corporation, Tokyo, Japan.). In the present patient, the right kidney was also removed due to a large cyst in the kidney.

The postoperative recovery was uneventful, with the exception of a grade-A pancreatic fistula, which healed without intervention 5 days after surgery. The present patient was discharged 13 days after surgery with partially controlled symptoms. At 2 months after surgery (the last follow-up), the symptom of upper abdominal pain was completely absent and appetite was improved, along with a weight gain of $3 \mathrm{~kg}$. Endoscopic examination indicated marked improvement of duodenal lesions, including disappearance of mucosal edema and reduction of polypoid protuberances. Imaging data are not available since the patient did not accept CT or MRI investigation.

\section{Discussion}

The exact pathogenesis of GP has not been clearly identified to date, although various hypotheses have been suggested $(4,11,12)$. Furthermore, a specific treatment strategy for GP has yet to be established (4). Different types of treatment have been reported, including conservative therapy, endoscopic treatment and surgery. Conservative treatment options for GP include abstinence from alcohol and tobacco, recovery of pancreatic function and analgesics $(3,4,7,12)$; however, typically these are only temporarily effective (12). Therefore, medical and endoscopic therapy are frequently regarded as 

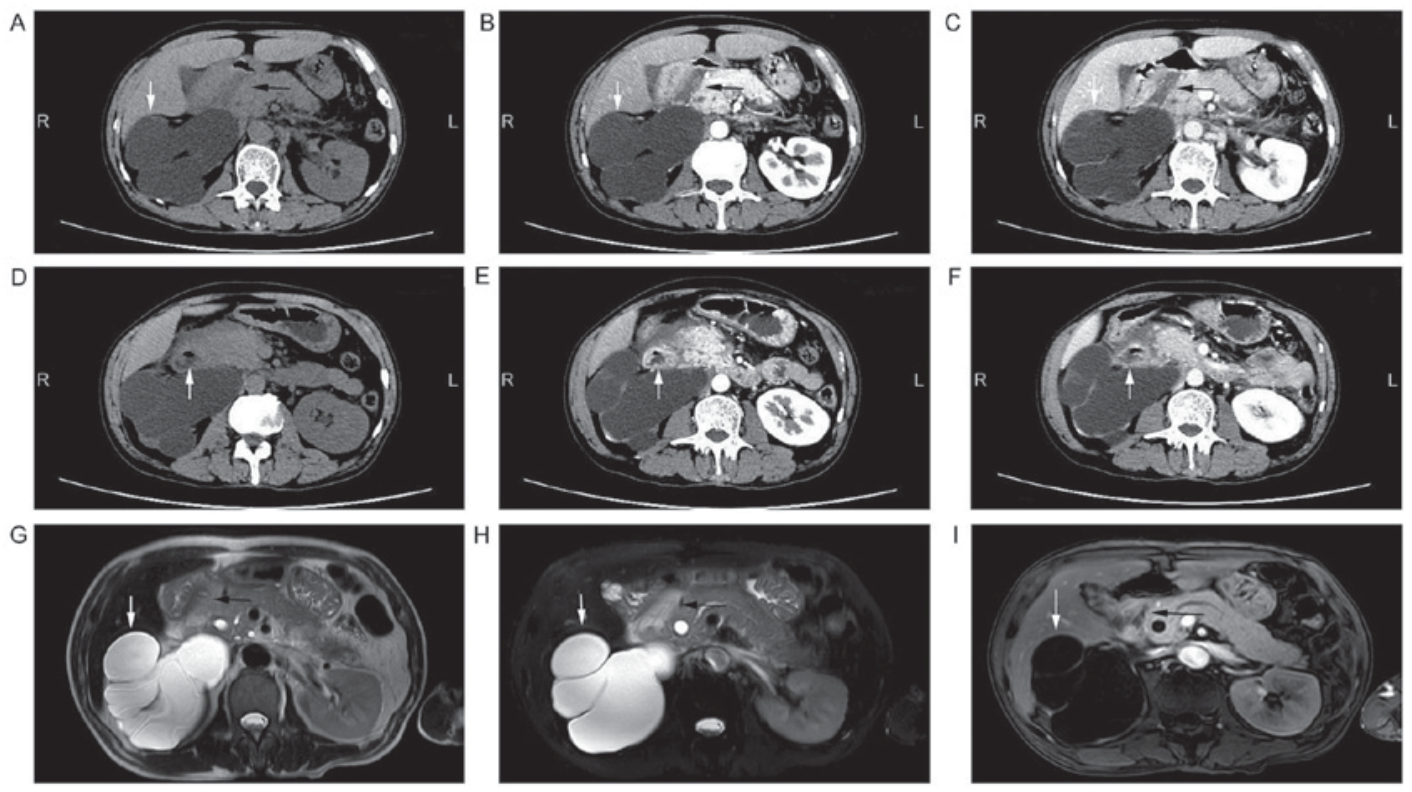

Figure 1. Imaging examination of the abdomen. CT images of the abdomen presented as (A) a plain scan, (B) arterial phase of contrast-enhanced scan and (C) venous phase of contrast-enhanced scan. A hypodense lesion (black arrow) was observed in the groove area, and a large cyst was shown in the right kidney (white arrow). CT images of the abdomen presented as (D) a plain scan, (E) arterial phase of contrast-enhanced scan and (F) venous phase of contrast-enhanced scan, showed thickening duodenal wall and cystic formation (white arrow) on the second part of the duodenum. (G) Plain T2-weighted, (H) fat-suppressed T2-weighted and (I) contrast-enhanced T1-weighted MRI scans of the abdomen are shown. A lesion (black arrow) was detected in the groove area in the T1-weighted (hypointense) and T2-weighted (hyperintense) images. A large cyst of right kidney (white arrow) was also identified. CT, computed tomography; MRI, magnetic resonance imaging.

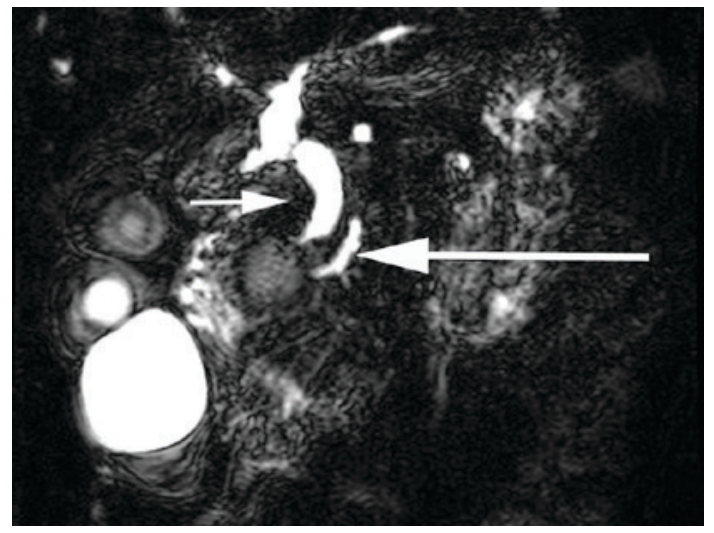

Figure 2. MRCP image, demonstrating that the proximal common bile duct was mildly dilated and the distal bile duct was slightly narrow (small arrow). Mildly dilated main pancreatic duct was also presented (large arrow). Accessory pancreatic duct was not demonstrated on the MRCP image. MRCP, magnetic resonance cholangiopancreatography.

reasonable treatment options prior to surgical treatment (13). Surgery is usually considered as the final choice when the symptoms are not improved by other measures, or in cases with a suspicion of malignancy (14). PD is the main surgical option for GP at present, since it can provide an effective and permanent outcome $(6,15)$. This procedure includes the resection of the pancreatic head, duodenum, distal bile duct and complicated digestive tract reconstruction. Thus, $\mathrm{PD}$ is considered to be an invasive procedure for GP treatment. DPPHR has also been performed in certain cases and has achieved satisfactory results of symptom relief $(3,4,8)$. Another technique is PPDR, which is particularly suitable for cases with significant duodenal involvement. A gastroenteroanastomosis
A
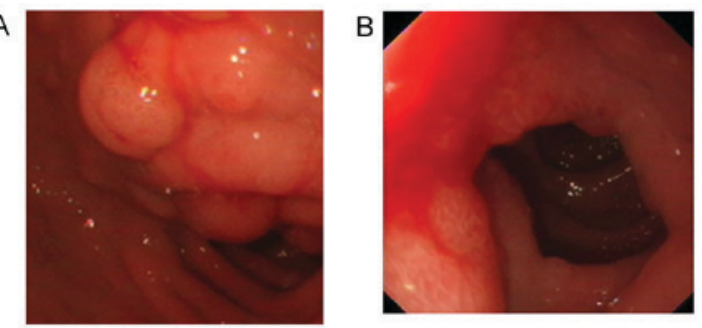

C

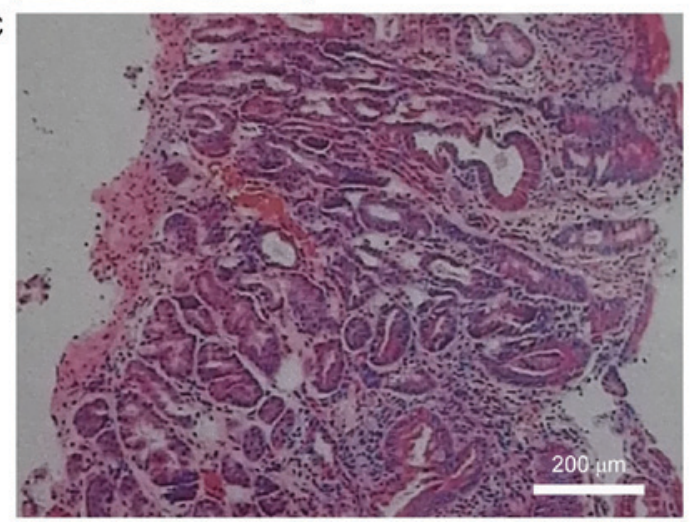

Figure 3. Endoscopic examination. (A) Edematous mucosa and multiple polypoid protuberances (white arrow) were shown in the first and second portions of the duodenum. (B) No luminal narrowing of duodenum was identified by endoscopy. (C) Histological examination of the polypoid biopsy specimen of duodenum indicated chronic and active mucosal inflammation and edema in the mesenchyme (hematoxylin and eosin staining; magnification, $\mathrm{x} 400)$.

may be a choice for patients with significant duodenal stenosis but without intractable pain (10), or in patients unsuitable for PD (14). 
A

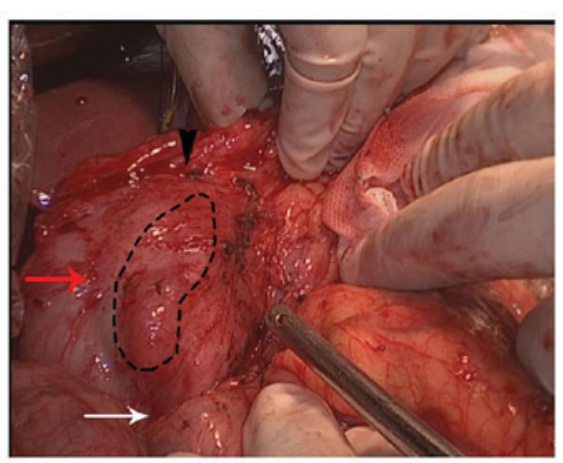

C

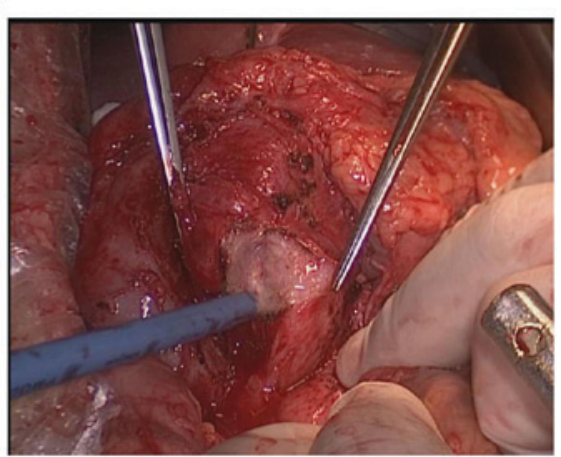

E

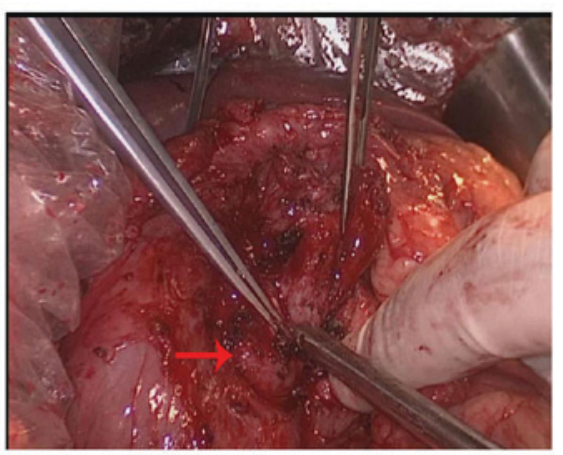

B

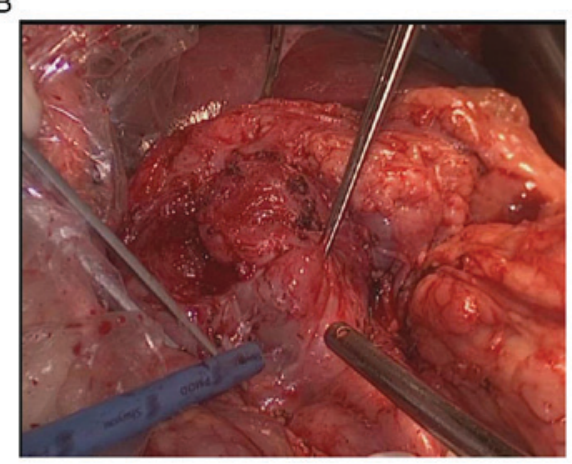

D

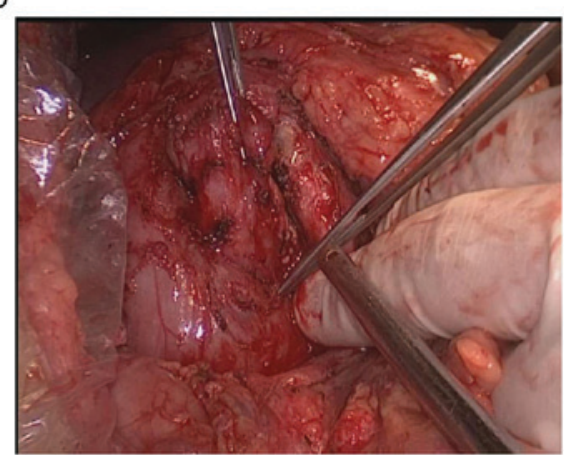

$\mathrm{F}$

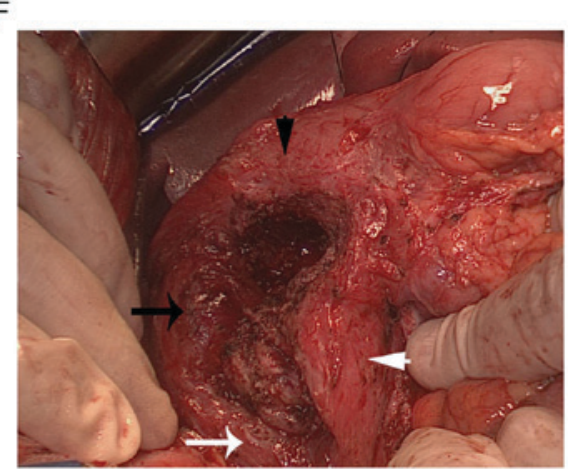

Figure 4. Intraoperative pictures. (A) The groove area (black dotted line) was observed as a narrowing area with groove-like shape in the right part of the pancreatic head. The first, second and third portion of the duodenum are shown by the black triangle, black arrow and white arrow, respectively. (B) Loose connective tissues and blood vessels between the pancreatic head and duodenum were dissociated. (C) Pancreatic tissue was divided along the left side of the groove area, preserving the pancreatic tissue between the superior mesenteric vein and the groove. (D) The inferior part of the groove pancreas was lifted upward, and the division was continued along the posterior border of the groove. (E) The distal bile duct (DBD; red arrow) was observed. The separation of the posterior was along the front surface of the DBD. (F) Following resection and removal of the groove pancreas, a defect in the shape of a narrowing groove was shown. The first, second and third portion of the duodenum are shown by the black triangle, black arrow and white arrow, respectively, while the left part of pancreatic head is indicated by the white triangle.

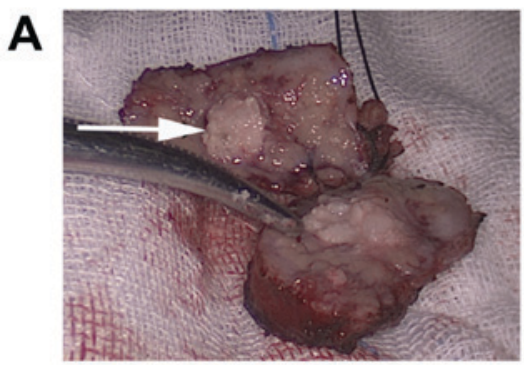

B

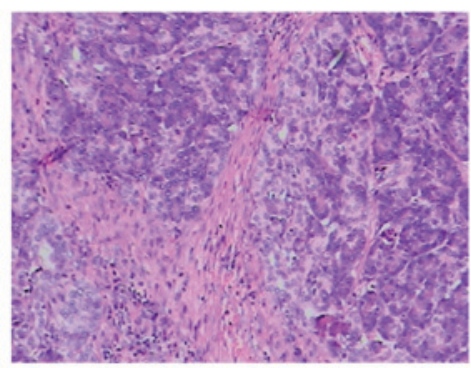

Figure 5. Pathological examination of the resected tissue. (A) Gross findings of the specimen. The resected specimen was approximately $5 \times 3 \mathrm{~cm}$ in size, and presented inflammatory fibrotic tissues in the groove area. A white mass (white arrow) was observed with a size of $2 \times 2.5 \mathrm{~cm}$ and a clear boundary. (B) Pathological examination of the specimen indicated acute and chronic inflammatory cell infiltration, and evident fibrosis of the pancreatic mesenchyme in the groove area (hematoxylin and eosin staining; magnification, magnification, x400). No calcification or protein plugs was observed. 
In the present study, a male patient with typical GP was treated with a novel surgical procedure, which involved the resection of the groove area of the pancreas only, while the duodenum, common bile duct, main pancreatic duct and the majority of the pancreatic head were preserved. This procedure is named as GRPH herein. To the best of our knowledge, the present study is the first report on GRPH for the surgical treatment of GP. Full pain control and weight gain were achieved at 2 months after surgery, while endoscopic examination indicated marked improvement of the duodenal lesions. The outcome of the current patient demonstrates that GRPH is a feasible and effective technique, and this procedure may be an alternative for the surgical treatment of GP without duodenal stenosis.

GRPH was performed for the patient in the present study based on the following considerations: The characteristics of clinical manifestations of the patient included recurrent upper abdominal pain, poor appetite and a weight loss. The cardinal symptom was abdominal pain, and the main aim in the reported case was to treat this symptom. Conservative treatments were not effective, and endoscopic therapy was not adopted since no minor papilla was identified and there were no evidence of ductal obstruction. Therefore, surgical treatment was then considered. Duodenum-preserving surgery was adopted in the present case, since there was no luminal stenosis or significant deformation of duodenum. Furthermore, the root cause of the abdominal pain in GP remains unclear, although it appears to be multifactorial and various hypotheses have been raised $(4,11,12,16)$. The anatomical or functional obstruction of the minor papilla is considered to be a possible cause of pain $(3,16,17)$. However, in the patient of the present study, minor papilla or an accessory duct were not identified. Parenchymal inflammation is also considered as another important factor for the development of pain (16). Inflamed pancreatic tissues produce numerous inflammatory mediators, which stimulate the sensory nerves, resulting in pain $(16,17)$. In GP, the most significant lesion of the pancreas is found in the groove area of the pancreatic head. The groove area is likely to be the inflammatory focus (16). From the pathological point of view, this lesion in the groove area consists of inflammatory, atrophic, fibrotic and nonfunctional pancreatic tissues (16). However, the pancreatic head has been proposed to be the pacemaker of GP (18). In the current study, we hypothesized that the groove area of pancreatic head may serve as the trigger point of abdominal pain in GP. Thus, resection of the groove, rather than the entire pancreatic head, may ease the release of inflammatory mediators and subsequently improve the abdominal pain, helping to improve the progress of GP. Subsequent to GRPH, full pain control and a weight gain were achieved 2 months after surgery, and endoscopic examination indicated marked improvement of the duodenal lesions. This satisfactory result verified our hypothesis, and therefore GRPH may be an alternative method for the surgical treatment of GP without duodenal stenosis. Finally, although the groove area is regarded as the anatomic space between the dorsocranial part of the pancreas head, the duodenum and the common bile duct, the groove area is an integral part of the pancreatic head and it cannot be easily detached from the pancreas, based on previous surgical experience. Therefore, the surgery performed in the present study was termed as 'groove resection of the pancreatic head'.

GRPH should be considered when the conservative treatment is not effective, and there are certain indications, including chronic inflammation mainly concentrated in the groove area with no evident pathological changes in other positions, no significant duodenal stenosis and no significant biliary obstruction. The contraindications include the presence of parts other than the groove area with apparent pancreatic lesions, complicated with duodenum and/or biliary obstruction. GRPH has the advantage of minor injury, organ preservation and avoiding the removal of various tissues and organs. However, GRPH cannot replace the PD and DPPHR entirely, since GRPH applies only to certain cases (refer to the aforementioned indications for GRPH). In addition, PD or DPPHR are considered when GRPH is not an option.

In conclusion, avoiding multi-organ resection, such as that performed during the PD procedure, is the greatest advantage of GRPH. In the surgery conducted in the present study, only the groove area was resected, while the duodenum, the main pancreatic duct and the majority of the pancreatic head were preserved. The absence of biliary or pancreatic duct reconstruction is another advantage of the GRPH procedure. The indication of GRPH mainly includes GP with severe lesions on the groove area. The contraindications include severe lesions and stenosis of the duodenum, and significant bile or pancreatic duct obstruction. However, further follow-up is required in the present case to observe the long-term effects of GRPH.

\section{Acknowledgements}

The present study was supported by the Social Development Project of Jiangsu Science Department (grant no. BE2016658).

\section{References}

1. Hernández-Jover D, Pernas JC, González-Ceballos S, Lupu I, Monill JM and Pérez C: Pancreatoduodenal junction: Review of anatomy and pathologic conditions. J Gastrointest Surg 15: 1269-1281, 2011

2. Becker V: Bauchspeicheldrüse; In Doerr W, Seifert G, Uhlinger E (eds): Spezielle pathologische Anatomie. Vol 4. Berlin, Springer, 1973.

3. Tezuka K, Makino T, Hirai I and Kimura W: Groove pancreatitis. Dig Surg 27: 149-152, 2010.

4. Egorov VI, Vankovich AN, Petrov RV, Starostina NS, Butkevich ATs, Sazhin AV and Stepanova EA: Pancreaspreserving approach to 'paraduodenal pancreatitis' treatment: Why, when, and how? Experience of treatment of 62 patients with duodenal dystrophy. Biomed Res Int 2014: 185265, 2014

5. Arvanitakis M, Rigaus J, Toussaint E, Eisendrath P, Bali MA, Matos C, Demetter P, Loi P, Closset J, Deviere J and Delhaye M: Endotherapy for paraduodenal pancreatitis: A large retrospective case series. Endoscopy 46: 580-587, 2014.

6. Rahman SH, Verbeke CS, Gomez D, McMahon MJ and Menon KV: Pancreatico-duodenectomy for complicated groove pancreatitis. HPB (Oxford) 9: 229-234, 2007.

7. Manzelli A, Petrou A, Lazzaro A, Brennan N, Soonawalla Z and Friend P: Groove pancreatitis. A mini-series report and review of literature. JOP 12: 230-233, 2011.

8. German V, Ekmektozoglou KA, Kyriakos N, Patouras P and Kikilas A: Pancreatitis of the gastroduodenal groove: A case report. Case Report Med 2010: 329587, 2010.

9. Patriti A, Castellani D, Partenzi A, Carlani M and Casciola L: Pancreatic adenocarcinoma in paraduodenal pancreatitis: A note of caution for conservative treatments. Updates Surg 64: 307-309, 2012. 
10. Rebours V, Lévy P, Vullierme MP, Couvelard A, O'Toole D, Aubert A, Palazzo L, Sauvanet A, Hammel P, Maire F, et al: Clinical and morphological features of duodenal cystic dystrophy in heterotopic pancreas. Am J Gastroenterol 102: 871-879, 2007.

11. Di Sebastiano P, di Mola FF, Buchler MW and Friess H: Pathogenesis of pain in chronic pancreatitis. Dig Dis 22: 267-272, 2004

12. Levenick JM, Gordon SR, Sutton JE, Suriawinata A and Gardner TB: A comprehensive, case-based review of groove pancreatitis. Pancreas 38: e169-e175, 2009.

13. Casetti L, Bassi C, Salvia R, Butturini G, Graziani R, Falconi M, Frulloni L, Crippa S, Zamboni G and Pederzoli P: 'Paraduodenal' pancreatitis: Results of surgery on 58 consecutives patients from a single institution. World J Surg 33: 2664-2669, 2009.

14. Pallisera-Lioveras A, Ramia-Angel JM, Vicens-Arbona $C$ and Cifuentes-Rodenas A: Groove pancreatitis. Rev Esp Enferm Dig 107: 280-288, 2015.
15. Triantopoulou C, Dervenis C, Giannakou N, Papailiou J and Prassopoulos P: Groove pancreatitis: A diagnostic challenge. Eur Radiol 19: 1736-1743, 2009.

16. Strate T, Knoefel WT, Yekebas E and Izbicki JR: Chronic pancreatitis: Etiology, pathogenesis, diagnosis, and treatment. Int J Colorectal Dis 18: 97-106, 2003.

17. Anaparthy R and Pasricha PJ: Pain and chronic pancreatitis: Is it the plumbing or the wiring? Curr Gastroenterol Rep 10: 101-106, 2008.

18. Izbicki JR, Bloechle C, Knoefel WT, Rogiers X and Kuechler T: Surgical treatment of chronic pancreatitis and quality of life after operation. Surg Clin North Am 79: 913-944, 1999. 